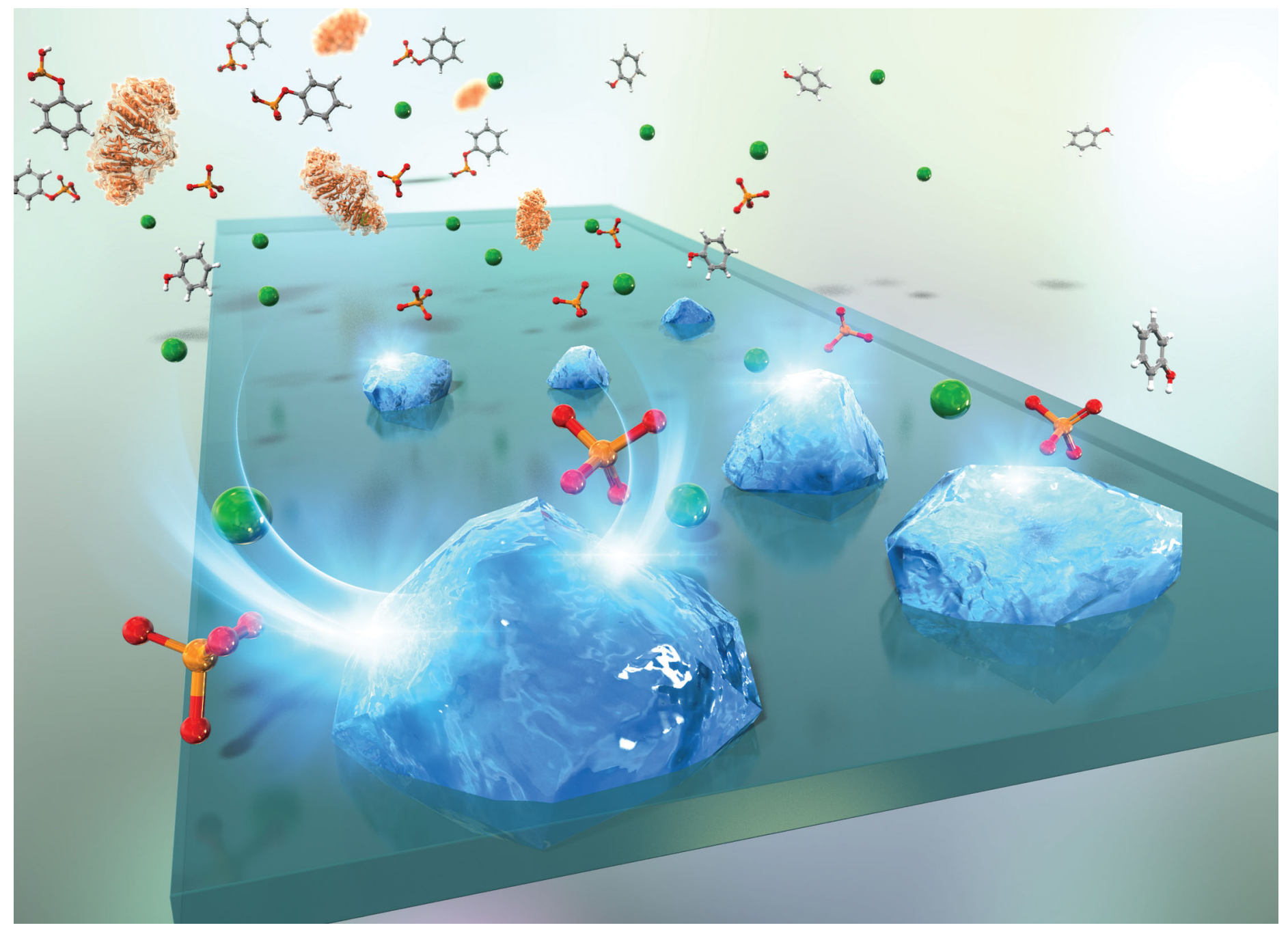

Showcasing research from Professor Kawashita's laboratory, Institute of Biomaterials and Bioengineering, Tokyo Medical and Dental University, Tokyo, Japan.

Behaviour of calcium phosphate ester salts in a simulated body fluid modified with alkaline phosphatase: a new concept of ceramic biomaterials

Ceramic biomaterials that promote bone regeneration are vital to the treatment of bone-degenerative diseases. Inspired by biomineralisation in our bones, salts of the calcium ion and phosphate esters (SCPE) are promising novel ceramic biomaterials showing a biomolecule-responsive function, because our bone tissues contain alkaline phosphatase. In this study, the behavior of calcium phenylphosphate (CaPP), a typical SCPE, under conditions mimicking an in vivo environment were investigated. The findings of this study demonstrate that CaPP is a good candidate for a new class of ceramic biomaterials that respond to trigger biomolecules.
As featured in:

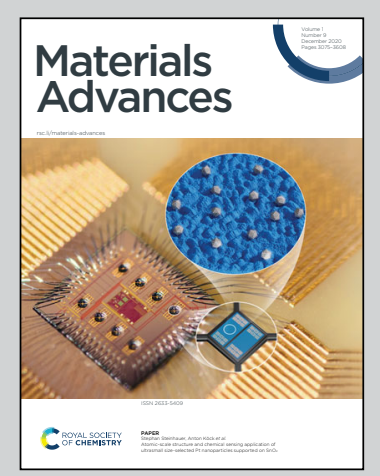

See Taishi Yokoi et al., Mater. Adv., 2020, 1, 3215. 
Check for updates

Cite this: Mater. Adv., 2020, 1, 3215

\title{
Behaviour of calcium phosphate ester salts in a simulated body fluid modified with alkaline phosphatase: a new concept of ceramic biomaterials
}

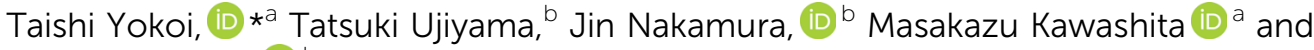 \\ Chikara Ohtsuki (D) ${ }^{\text {b }}$
}

\begin{abstract}
Ceramic biomaterials that promote bone tissue regeneration are vital in the treatment of various bonedegenerative diseases and have stimulated intense bioceramic-related research. Salts of the calcium ion and phosphate esters (SCPE) can be transformed into calcium phosphate in aqueous environments containing alkaline phosphatase (ALP). Inspired by the formation process of hydroxyapatite (HAp) in our bones, these SCPEs are promising novel ceramic biomaterials showing a biomolecule-responsive function for bone repairing applications, because our bone tissues contain ALP. In this study, the behaviour of calcium phenylphosphate (CaPP), a typical SCPE, under conditions mimicking an in vivo environment were investigated. A simulated body fluid (SBF) modified with ALP was used as reaction media. The transformation from CaPP to HAp in the SBF containing ALP occurred, while no reaction occurred without ALP present. The transformation process of CaPP can be concluded as follows: first, soaked CaPP dissolves into the SBF and forms calcium ions and phenylphosphate ions. Subsequently, phosphate ions are generated by hydrolysis of phenylphosphate ions. This hydrolytic reaction is mediated by ALP. Finally, HAp is formed by the reaction of calcium, phosphate, and hydroxide ions in the SBF. Interestingly, the transformation reaction of CaPP occurred under conditions outside the optimal $\mathrm{pH}$ ranges for ALP. The findings of this study demonstrate that SCPEs are good candidates for a new class of ceramic biomaterials that respond to trigger biomolecules, namely ALP, in this case.
\end{abstract}

Received 13th August 2020,

Accepted 29th October 2020

DOI: 10.1039/d0ma00600a

rsc.li/materials-advances

\section{Introduction}

Ceramic biomaterials are categorized into three types based on the body's hard tissue response: bioinert, bioactive, and biodegradable. ${ }^{1}$ Aluminium and zirconium oxides are typical examples of bioinert ceramics. Hydroxyapatite ( $\mathrm{HAp}, \mathrm{Ca}_{10}\left(\mathrm{PO}_{4}\right)_{6}$ $\left.(\mathrm{OH})_{2}\right)$ and tricalcium phosphate (TCP, $\left.\mathrm{Ca}_{3}\left(\mathrm{PO}_{4}\right)_{2}\right)$, which are often used for hard tissue regeneration, are typical bioactive and biodegradable ceramics, respectively. Many researchers in the field of ceramic biomaterials have tried to improve the performance of these bone repairing materials by controlling their microstructure ${ }^{2-4}$ or by adding other chemical species that can help promote bone formation and regeneration, such as silicon (Si). ${ }^{5-9}$ This direction of materials development is reasonable, and notable ceramic biomaterials have been

\footnotetext{
${ }^{a}$ Institute of Biomaterials and Bioengineering, Tokyo Medical and Dental University, 2-3-10 Kanda-Surugadai, Chiyoda-ku, Tokyo 101-0062, Japan. E-mail: yokoi.taishi.bcr@tmd.ac.jp

${ }^{b}$ Graduate School of Engineering, Nagoya University, Furo-cho, Chikusa-ku, Nagoya 464-8603, Japan
}

developed. However, the developed materials remain within this traditional framework. We believe that novel material designs are required to develop next-generation ceramic biomaterials.

Understanding the formation process of HAp in bone tissues is helpful for creating novel ceramic biomaterials. A brief explanation of this process is as follows: ${ }^{10}$ formation of HAp begins in matrix vesicles produced from osteoblasts. Then, as the HAp crystals penetrate the matrix vesicle membrane, they continue to grow before being deposited on the collagen fibrils. Alkaline phosphatase (ALP) plays a vital role in the crystal growth process of HAp because the phosphate ions produced in the hydrolysis of pyrophosphate by ALP are one source of phosphate used by HAp for crystal growth.

Inspired by the formation process of HAp in our bones, we suggest that salts of calcium ions and phosphate esters (SCPE) are good candidates for a novel ceramic biomaterial. SCPEs have been used as starting materials for calcium phosphate synthesis in artificial reaction systems. ${ }^{11-15}$ For example, HAp was formed from calcium phenylphosphate (CaPP), which is a type of SCPE, in aqueous solutions containing ALP. We expect that similar transformation reactions occur in vivo environments. 
After implantation of SCPEs into bone tissues, the salts dissolve and dissociate into calcium ions and phosphate esters. The phosphate esters are decomposed into phosphate and alcohol by ALP existing in our bones. The phosphate ions then react with calcium ions in the blood plasma, resulting in HAp formation. This sequence of chemical reactions resembles HAp growth in our bones. If these chemical reactions occur in vivo mediated by ALP, SCPEs can be newly categorized as 'bioresponsive' ceramic biomaterials.

We expect that SCPEs have potential as a novel class of ceramic biomaterials with biomolecule responsiveness for bone repairing. However, the behaviour of SCPEs in vivo is still unclear. The dissolution of SCPE and subsequent ALP mediated transformation into HAp are key processes that must be understood before application. To understand the key processes of SCPE, we fundamentally investigated the behaviour of SCPE in vitro. We used a simulated body fluid (SBF) modified with ALP as a reaction medium. The chosen SBF was developed by Kokubo and his colleague and has been used to estimate the behaviour of materials in bony defects. ${ }^{16}$

\section{Experimental}

\section{CaPP synthesis}

We used CaPP as a model SCPE because we expected it to show appropriate solubility behaviour, and its synthesis was as follows: calcium chloride $\left(\mathrm{CaCl}_{2}, 95.0 \%\right.$, Nacalai Tesque Inc., Kyoto, Japan) was dissolved in ultrapure water. The $\mathrm{pH}$ of the solution was adjusted to equal 10 by adding an ammonia solution $\left(\mathrm{NH}_{3}, 28 \%\right.$, Wako Pure Chemical Industries, Ltd, Osaka, Japan) at $37{ }^{\circ} \mathrm{C}$. The calcium chloride solution was transferred to a volumetric flask and ultrapure water was added to adjust the total volume of the solution. The concentration of calcium chloride in this solution was $50 \mathrm{~mol} \mathrm{~m}^{-3}$. Phenylphosphoric acid $\left(\mathrm{C}_{6} \mathrm{H}_{5} \mathrm{OPO}_{3} \mathrm{H}_{2}, 99.0 \%\right.$, Tokyo Chemical Industry Co., Ltd, Tokyo, Japan) was dissolved in ultrapure water. The $\mathrm{pH}$ of the solution was adjusted to 10 at $37^{\circ} \mathrm{C}$ with the aqueous ammonia. The phenylphosphate solution was transferred to a volumetric flask and ultrapure water was added to prepare a $50 \mathrm{~mol} \mathrm{~m}^{-3}$ phenylphosphate solution. The calcium chloride solution and phenylphosphate solution $\left(250 \mathrm{~cm}^{3}\right.$ of each) were mixed at $37{ }^{\circ} \mathrm{C}$ for 2 hours with $300 \mathrm{rpm}$ stirring. The formed precipitate was collected by vacuum filtration and washed with ultrapure water and ethanol. Subsequently, the precipitate was dried at $40{ }^{\circ} \mathrm{C}$ overnight.

\section{Soaking of samples in SBF}

SBF was prepared as follows: $700 \mathrm{~cm}^{3}$ of ultrapure water was added to a $1000 \mathrm{~cm}^{3}$ glass beaker and stirred with a magnetic stirrer. The reagent-grade chemicals given in Table 1 were then dissolved in the order listed. Each reagent was allowed to completely dissolve before the addition of the next reagent. The solution was maintained at $36.5{ }^{\circ} \mathrm{C}$ and the $\mathrm{pH}$ was adjusted to 7.4 by adding a $1.0 \mathrm{~mol} \mathrm{dm}^{-3}$ hydrochloric acid. After the $\mathrm{pH}$ adjustment, the solution was transferred to a
Table 1 Reagents used to prepare $1000 \mathrm{~cm}^{3}$ of the simulated body fluid

\begin{tabular}{lll}
\hline Order & Reagent & Amount \\
\hline 1 & $\mathrm{NaCl}$ & $7.996 \mathrm{~g}$ \\
2 & $\mathrm{NaHCO}$ & $0.350 \mathrm{~g}$ \\
3 & $\mathrm{KCl}$ & $0.224 \mathrm{~g}$ \\
4 & $\mathrm{~K}_{2} \mathrm{HPO}_{4} \cdot 3 \mathrm{H}_{2} \mathrm{O}$ & $0.228 \mathrm{~g}$ \\
5 & $\mathrm{MgCl}_{2} \cdot 6 \mathrm{H}_{2} \mathrm{O}$ & $0.305 \mathrm{~g}$ \\
6 & $1.0 \mathrm{~mol} \mathrm{dm}^{-3} \mathrm{HCl}$ & $40 \mathrm{~cm}^{3}$ \\
7 & $\mathrm{CaCl}_{2}$ & $0.278 \mathrm{~g}$ \\
8 & $\mathrm{Na}_{2} \mathrm{SO}_{4}$ & $0.071 \mathrm{~g}$ \\
9 & $\left(\mathrm{CH}_{2} \mathrm{OH}\right)_{3} \mathrm{CNH}_{2}$ & $6.057 \mathrm{~g}$ \\
\hline
\end{tabular}

volumetric flask and ultrapure water was added to adjust the total volume of the solution to $1000 \mathrm{~cm}^{3}$. All the reagents shown in Table 1, except for the $1.0 \mathrm{~mol} \mathrm{dm}^{-3}$ hydrochloric acid solution, were purchased from Nacalai Tesque, Inc. The $1.0 \mathrm{~mol} \mathrm{dm}{ }^{-3}$ hydrochloric acid solution was prepared by diluting a 35 mass\% hydrochloric acid solution ( $\mathrm{HCl}$, Wako Pure Chemical Industries Ltd).

Further, $100 \mathrm{mg}$ of CaPP powder was soaked in $25 \mathrm{~cm}^{3}$ of SBF with 0, 1.25, 2.5, and 7.5 units of ALP (from bovine intestinal mucosa, Sigma-Aldrich Japan Co., Tokyo, Japan) and maintained at $36.5{ }^{\circ} \mathrm{C}$ for 1,3 , and 7 days (d). ALP was added to the SBF at daily intervals owing to its gradual deactivation. Since the ALP content of normal serum falls in the range 20-140 units $\mathrm{dm}^{-3},{ }^{17}$ the amounts added to SBF in our experiments were set to cover the range of ALP activity in normal serum. After soaking, the sample powder was removed from the SBF by filtration. The extracted sample powder was rinsed with ultrapure water and ethanol and subsequently dried at $40{ }^{\circ} \mathrm{C}$. Hereafter, samples soaked in SBF are named $\operatorname{ALP} X . X(=0,1.25,2.5$, and 7.5) indicates the amount of ALP used for the soaking experiment.

\section{Characterization}

The morphology of the samples was observed using a scanning electron microscope (SEM; JSM5600, JEOL Ltd, Tokyo, Japan) after thin Au film coating. The crystalline phase of the samples was examined by powder X-ray diffraction (XRD; RINT PC2100, Rigaku Co., Tokyo, Japan) using $\mathrm{Cu} \mathrm{K} \alpha$ radiation. Chemical structures of the samples were characterized using a Fouriertransform infrared (FTIR) spectrometer (FT/IR-610, JASCO Co., Tokyo, Japan) by using the KBr tablet method. The mass ratio of the sample with respect to $\mathrm{KBr}$ was 1 mass\%.

The $\mathrm{pH}$ of the SBF after soaking the sample powder was measured by a glass-electrode type $\mathrm{pH}$ meter (D-51, Horiba Ltd, Kyoto, Japan). Concentrations of calcium and phosphorus in the SBF were measured by inductively coupled plasma atomic emission spectroscopy (Optima 2000DV, PerkinElmer Japan, Kanagawa, Japan).

\section{Results}

Morphological changes of samples after soaking in the SBF were investigated using SEM. Fig. 1 shows the morphology of samples before and after SBF soaking. The plate-shaped crystals with clear 

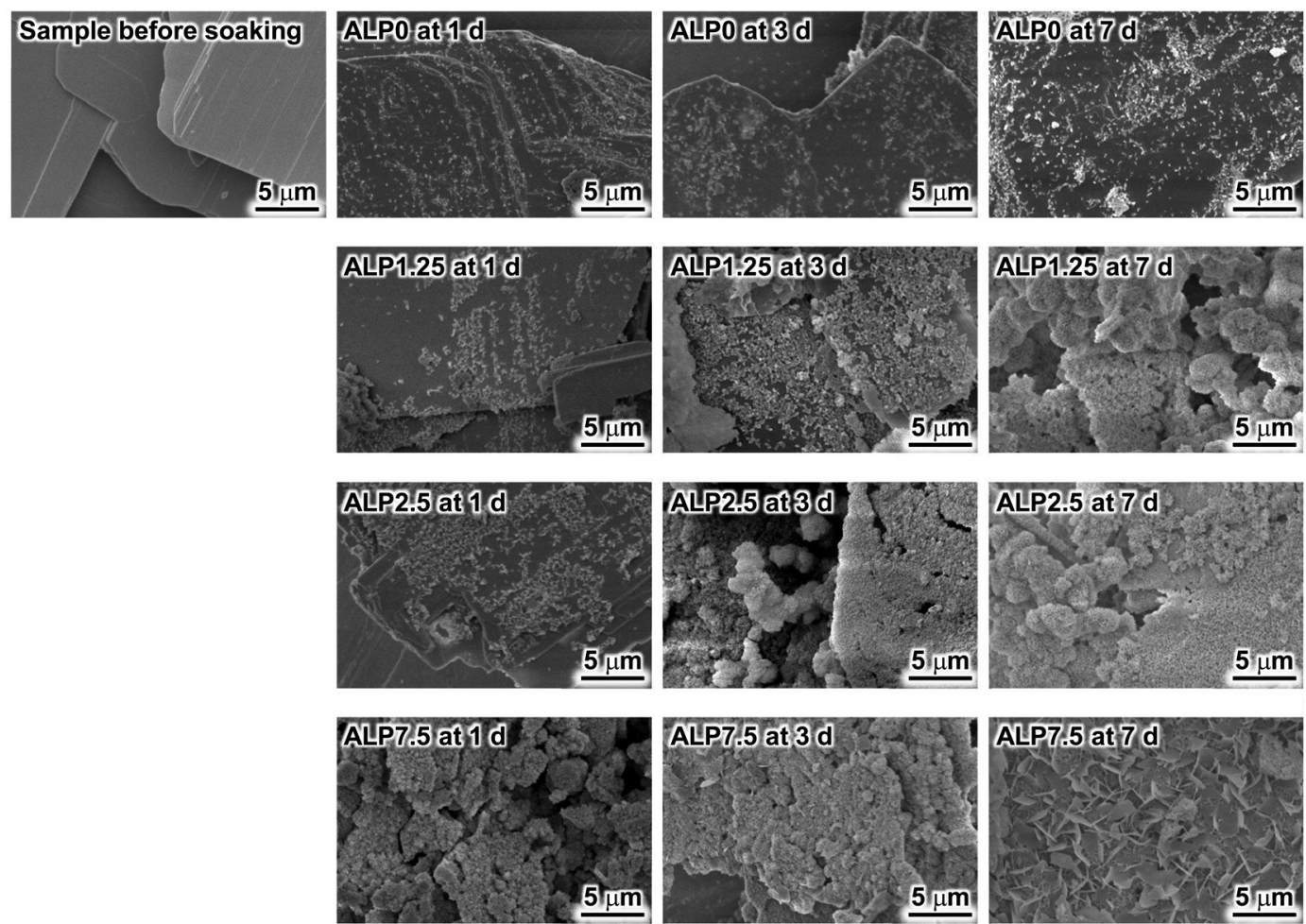

Fig. 1 SEM photographs of CaPP samples before and after soaking in SBF.

crystal facets were observed in the sample before soaking in SBF. Small precipitates were observed on the plate-shaped crystals in ALP0 after $1 \mathrm{~d}$. Additionally, the corners of the plate-shaped crystals were slightly rounded in this sample. Significant morphological changes after an extension of the soaking periods were not observed. Small precipitates were also observed on the plateshaped crystals in ALP1.25 after $1 \mathrm{~d}$. The number of small precipitates increased with longer soaking periods. Agglomerated precipitates with a spherical shape were observed in ALP1.25 at $7 \mathrm{~d}$. Small precipitates were also observed on the plate-shaped crystals in ALP2.5 after $1 \mathrm{~d}$. The small precipitates almost completely covered the surface of the plate-shaped crystals at $3 \mathrm{~d}$. Spherical agglomerates were observed in ALP2.5 at 7 d. In ALP7.5 at $1 \mathrm{~d}$ and $3 \mathrm{~d}$, many small precipitates were observed. In addition to thin petal-like crystals, small precipitates were observed in ALP7.5 at $7 \mathrm{~d}$.

Changes to the crystalline phase of the samples after soaking in SBF were examined by powder XRD, as shown in Fig. 2 . Reflection peaks detected in the sample before soaking in SBF were assigned to CaPP. No other crystalline phases were detected in the sample. The reflection peak assignment of CaPP was conducted based on previous reports. ${ }^{18}$ The reflection peak intensities of CaPP decreased with longer soaking periods in both ALP0 and ALP1.25 samples. Only CaPP was detected in ALP0 samples up to $7 \mathrm{~d}$ of soaking, while very weak reflection peaks derived from HAp (powder diffraction file \#00-009-0432) were detected around $26^{\circ}$ and $31^{\circ}$ in ALP1.25 at $7 \mathrm{~d}$, in addition to the strong reflection peaks derived from remaining CaPP. In the case of the ALP2.5 samples, the reflection peak intensities
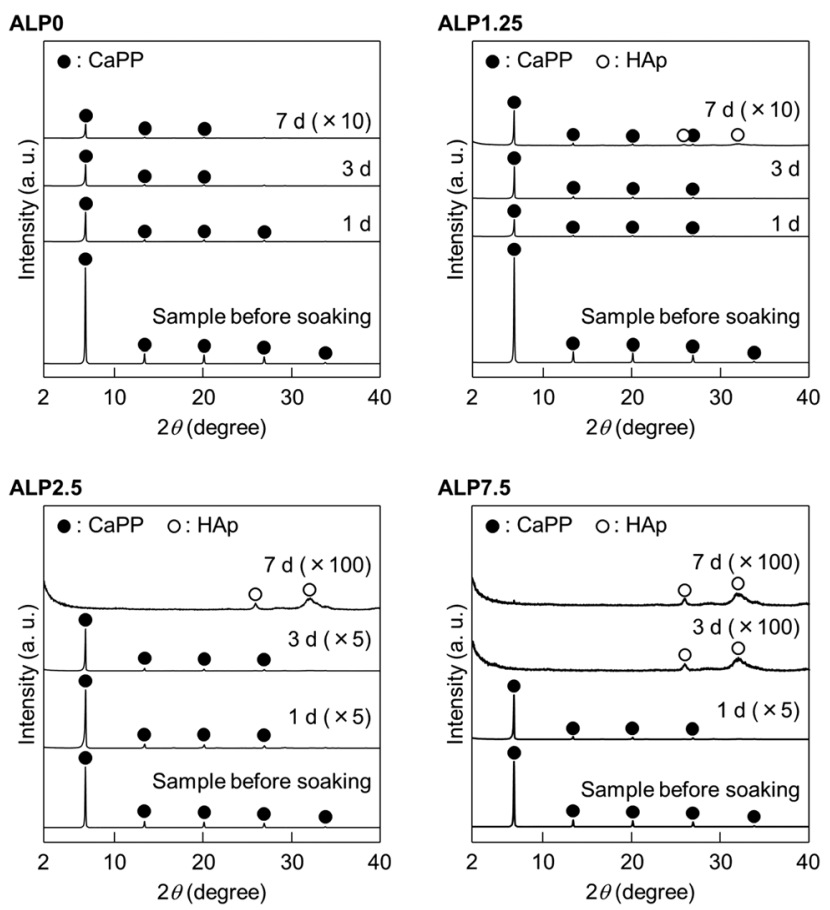

Fig. 2 Powder XRD patterns of CaPP samples before and after soaking in SBF.

derived from CaPP decreased with longer soaking periods. CaPP reflection peaks disappeared, and HAp signals were detected in ALP2.5 after $7 \mathrm{~d}$. In ALP7.5 at $1 \mathrm{~d}$, the reflection 


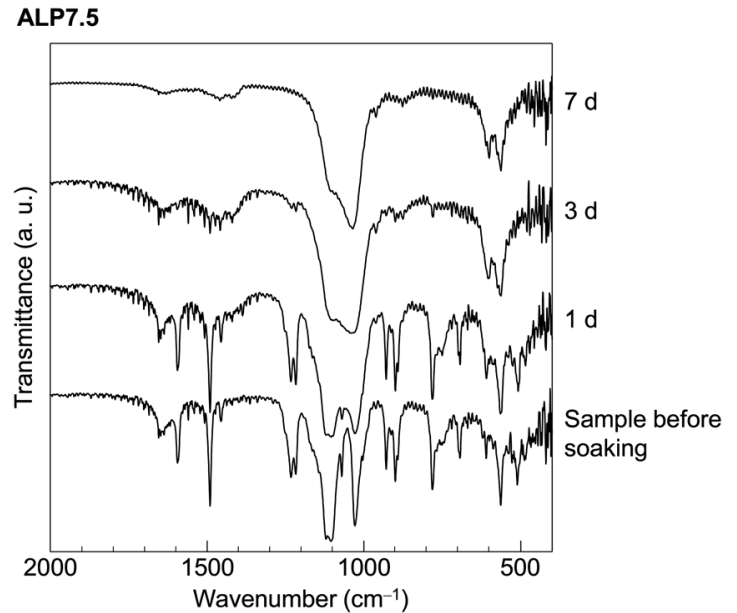

Fig. 3 FTIR spectra of ALP7.5 samples before and after soaking in SBF.

peak intensities of CaPP were decreased by soaking in SBF. The reflection peaks of CaPP disappeared in ALP7.5 at $3 \mathrm{~d}$ and $7 \mathrm{~d}$, while reflection peaks derived from HAp were detected in these samples.

FTIR spectra of ALP7.5 are shown in Fig. 3 as a representative sample of the chemical changes that occur by soaking in the SBF. Absorption peaks in the sample before soaking in SBF are assigned to CaPP based on previous reports. ${ }^{18}$ The peaks detected at around 1590 and $1490 \mathrm{~cm}^{-1}$ were derived from the ring stretching vibration mode of the phenyl group. These characteristic absorption peaks were detected in the samples before soaking in SBF and after soaking in SBF for $1 \mathrm{~d}$. While these absorption peaks were not detected in ALP2. 5 at $3 \mathrm{~d}$ and $7 \mathrm{~d}$, the absorption peaks in these samples appear to be derived from HAp. ${ }^{18,19}$

Time dependent changes to $\mathrm{Ca}$ and $\mathrm{P}$ concentrations in the SBF are shown in Fig. 4. The Ca concentration increased significantly to $8-10 \mathrm{~mol} \mathrm{~m}^{-3}$ after $1 \mathrm{~d}$ in all conditions. Subsequently, the Ca concentrations of ALP0 and ALP1.25 samples remained constant up to $7 \mathrm{~d}$. While the Ca concentrations of the ALP2.5 and ALP7.5 samples decreased with extended soaking periods. The Ca concentrations of ALP2.5 and ALP7.5 decreased to 6 and $1 \mathrm{~mol} \mathrm{~m}^{-3}$, respectively. The $\mathrm{P}$ concentration increased significantly to between $8-9 \mathrm{~mol} \mathrm{~m}^{-3}$
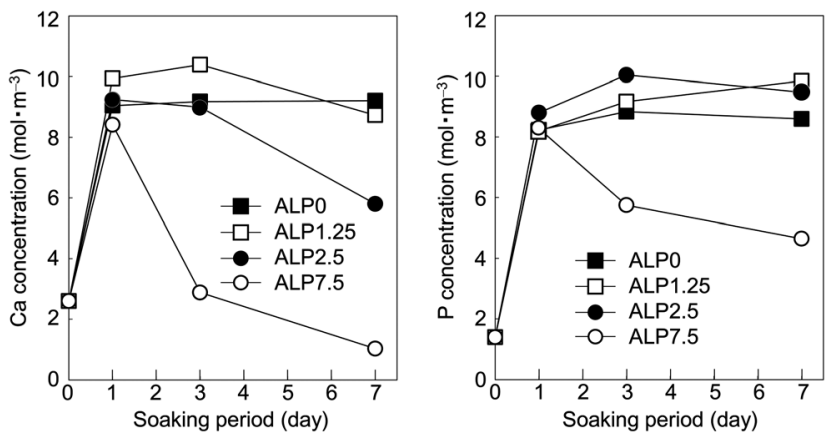

Fig. 4 Time dependent changes in Ca (left) and P (right) concentrations in SBF.

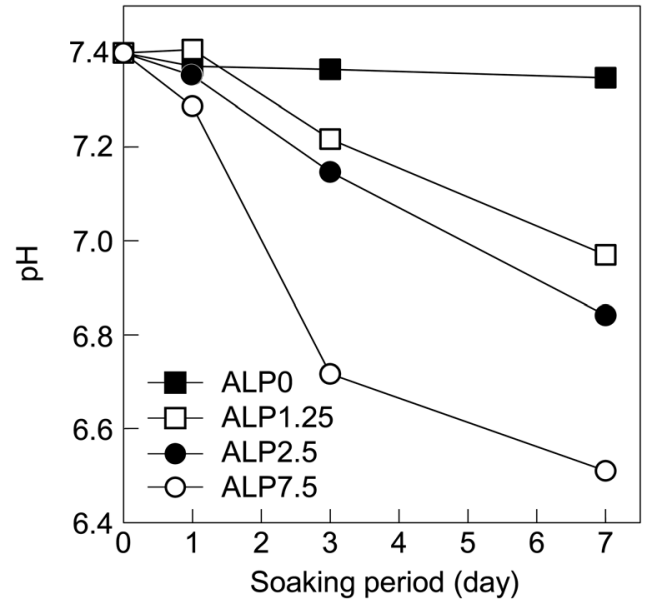

Fig. 5 Time dependent changes in SBF pH values.

after $1 \mathrm{~d}$ in all conditions. Subsequently, the P concentrations of the ALP0, ALP1.25, and ALP2.5 samples remained mostly constant up to $7 \mathrm{~d}$. In contrast, the $\mathrm{P}$ concentration of the ALP7.5 sample decreased after long soaking periods, reducing to $5 \mathrm{~mol} \mathrm{~m}^{-3}$ at $7 \mathrm{~d}$.

Time dependent changes in the SBF pH are shown in Fig. 5 . The $\mathrm{pH}$ values of the ALP0 sample was relatively constant at around 7.4. The $\mathrm{pH}$ values of ALP1.25 samples decreased after $3 \mathrm{~d}$, reaching 7 after $7 \mathrm{~d}$. ALP2.5 samples also saw a pH decrease after $3 \mathrm{~d}$, dropping to around 6.9 at $7 \mathrm{~d}$. The largest decrease in $\mathrm{pH}$ occurred in the ALP7.5 sample, which was measured to be 6.5 after $7 \mathrm{~d}$.

\section{Discussion}

\section{Transformation of CaPP in SBF}

The behaviour of CaPP in SBF without ALP was quite different from experiments where ALP was added to the SBF. The corners of CaPP crystals found in ALP0 at $1 \mathrm{~d}$ were slightly rounded after soaking in SBF (Fig. 1). Additionally, the $\mathrm{Ca}$ and $\mathrm{P}$ concentrations in SBF after exposure to ALP0 increased after $1 \mathrm{~d}$ (Fig. 4). These results indicate the dissolution of CaPP into the SBF, releasing calcium and phenylphosphate ions into solution. The degree of supersaturation of calcium phosphate was increased as more calcium ions were released in the SBF. The small precipitates identified by SEM observation were likely calcium phosphate precipitated from the SBF (Fig. 1). The crystalline phase of the small precipitates was difficult to identify by XRD (Fig. 2) because the amount of the tiny precipitate was too small for analysis. Morphological changes from extending the soaking period were hardly observed in ALP0 (Fig. 1). Additionally, Ca and P concentrations (Fig. 4) and $\mathrm{pH}$ values (Fig. 5) in the SBF were almost constant during the reaction periods of 1-7 d. These results suggest that calcium and phenylphosphate ion concentrations reached a saturation point and the system reached a steady-state after $1 \mathrm{~d}$. Hence, we conclude that CaPP does not transform into calcium phosphates, such as HAp, in SBF without ALP. 
Although the transformation of CaPP was not detected in ALP0 samples by XRD, HAp was detected in all ALP-added samples, namely ALP1.25, ALP2.5, and ALP7.5 (Fig. 2), especially in the ALP2.5 and ALP7.5 samples, where CaPP was completely transformed into HAp (Fig. 2 and 3). These results clearly indicate that HAp formation is mediated by ALP, even though the solution $\mathrm{pH}$ was outside of optimum conditions for ALP. The compositional formula of CaPP is assumed to be $\mathrm{Ca}\left(\mathrm{C}_{6} \mathrm{H}_{5} \mathrm{OPO}_{3}\right)$; thus, the chemical transformation from $\mathrm{CaPP}$ to HAp can be expressed as follows:

$$
\begin{aligned}
& 10 \mathrm{Ca}\left(\mathrm{C}_{6} \mathrm{H}_{5} \mathrm{OPO}_{3}\right)+12 \mathrm{H}_{2} \mathrm{O} \rightarrow \mathrm{Ca}_{10}\left(\mathrm{PO}_{4}\right)_{6}(\mathrm{OH})_{2}+4 \mathrm{H}_{3} \mathrm{PO}_{4}+ \\
& 10 \mathrm{C}_{6} \mathrm{H}_{5} \mathrm{OH}
\end{aligned}
$$

Time dependent changes of $\mathrm{Ca}$ and $\mathrm{P}$ concentrations and $\mathrm{pH}$ in the SBF (shown in Fig. 4 and 5) are helpful for understanding the behaviours of CaPP in the reaction solution. We focus on ALP7.5 samples as a representative to explain the detailed transformation behaviour of CaPP. After $1 \mathrm{~d}$, the $\mathrm{Ca}$ and $P$ concentrations in ALP7.5 increased due to the dissolution of CaPP. On $3 \mathrm{~d}$ or after, the $\mathrm{Ca}$ and $\mathrm{P}$ concentrations and pH decreased significantly in ALP7.5 samples. HAp formation was detected in these samples starting from $3 \mathrm{~d}$ by XRD (Fig. 2). Clearly, the decreases in $\mathrm{Ca}$ and $\mathrm{P}$ concentrations and $\mathrm{pH}$ is due to HAp formation through the following chemical equation.

$$
10 \mathrm{Ca}^{2+}+6 \mathrm{PO}_{4}^{3-}+2 \mathrm{OH}^{-} \rightarrow \mathrm{Ca}_{10}\left(\mathrm{PO}_{4}\right)_{6}(\mathrm{OH})_{2}
$$

The $\mathrm{P}$ concentration in the SBF after 1-7 $\mathrm{d}$ was the sum of concentrations of orthophosphate and phenylphosphate species. The initial orthophosphate concentration in the SBF was $1 \mathrm{~mol} \mathrm{~m}^{-3}$. The $\mathrm{P}$ concentration increased to $8.3 \mathrm{~mol} \mathrm{~m}^{-3}$ at $1 \mathrm{~d}$ before decreasing to $4.6 \mathrm{~mol} \mathrm{~m}^{-3}$ at $7 \mathrm{~d}$ in the ALP7.5 samples. Hence, at least $2.7 \mathrm{~mol} \mathrm{~m}^{-3}$ phenylphosphate was decomposed to orthophosphate and phenol by ALP, and the formed orthophosphate was consumed to form HAp. These findings provide evidence that hydrolysis of phenylphosphate by ALP occurs in SBF, and resulting orthophosphate produced from the hydrolysis reaction is used as a phosphate source for HAp formation.

Based on the above discussion, we can summarize the behaviour of CaPP in SBF containing ALP. When CaPP was dissolved in the SBF, calcium and phenylphosphate ions were released into solution. Then, phosphate ions were generated through the hydrolysis of phenylphosphate ion by ALP. Finally, HAp was formed from the reaction of calcium, phosphate, and hydroxide ions. The sequence of HAp formation from CaPP is schematically illustrated in Fig. 6.

\section{Effects of ALP concentrations on transformation rate of CaPP}

According to the results of SEM observation and XRD analysis (Fig. 1 and 2), the small precipitates formed on the plateshaped crystals were most likely HAp. SEM images imply that the formation rate of the HAp crystals increased with higher ALP concentration in the SBF. To clarify the effects of ALP concentration on the transformation rate of CaPP, time dependent changes to HAp fraction were calculated semiquantitatively from the integration of XRD reflection peak intensities of HAp and CaPP through the following equation:

$$
F_{\mathrm{HAp}}=I_{\mathrm{HAp}} /\left(I_{\mathrm{HAp}}+I_{\mathrm{CaPP}} / 49.5\right) \times 100
$$

where, $F_{\mathrm{HAp}}, I_{\mathrm{HAp}}$, and $I_{\mathrm{CaPP}}$ are a fraction of HAp with respect to CaPP in the sample, the integrated intensity of the 002 reflection peak of HAp detected at around $26^{\circ}$, and the integrated intensity of the strongest reflection peak of CaPP detected at around $7^{\circ}$, respectively, and 49.5 is a compensation coefficient. Fig. 7 shows the time dependent changes of $F_{\text {HAp }}$ calculated from the XRD patterns of the samples. $F_{\text {HAp }}$ values of ALP0, ALP1.25, and ALP2.5 were almost the same after $1 \mathrm{~d}$, while the ALP7.5 value was higher. This corresponds to the morphological observation results (Fig. 1). Additionally, time dependent changes of $F_{\text {HAp }}$ qualitatively agree with the transformation reaction progress estimated from morphological observations. The transformation rate from CaPP to HAp increased with ALP

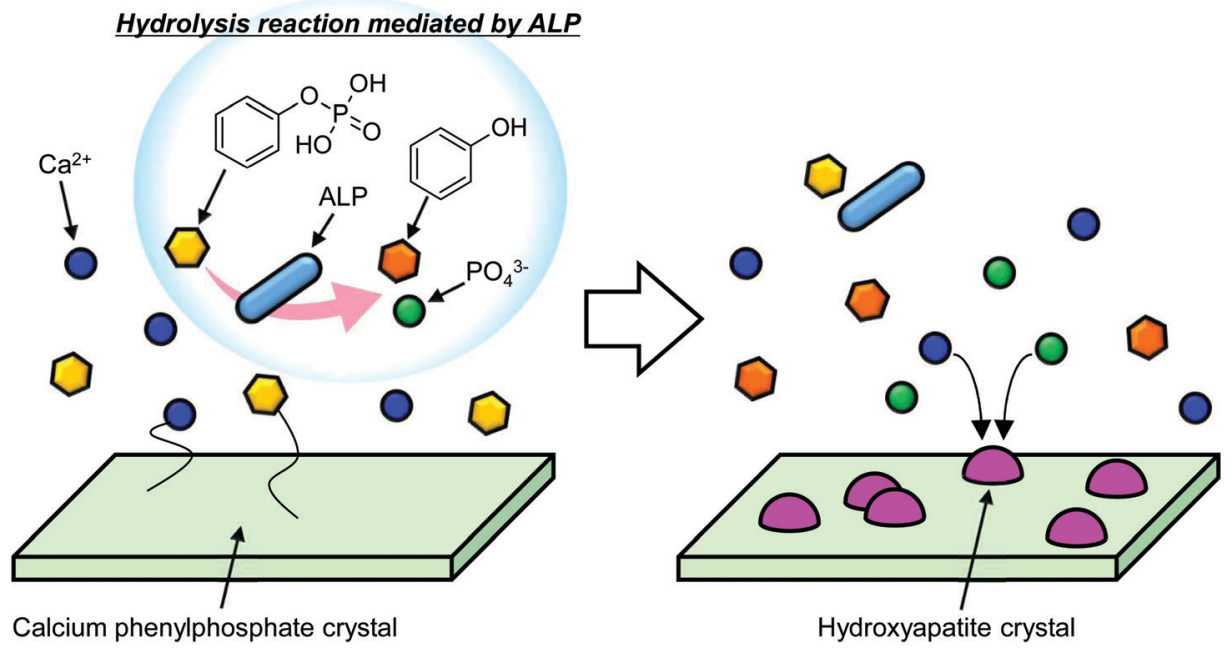

Fig. 6 Schematic illustration of transformation reaction of CaPP to HAp in SBF modified with ALP. 


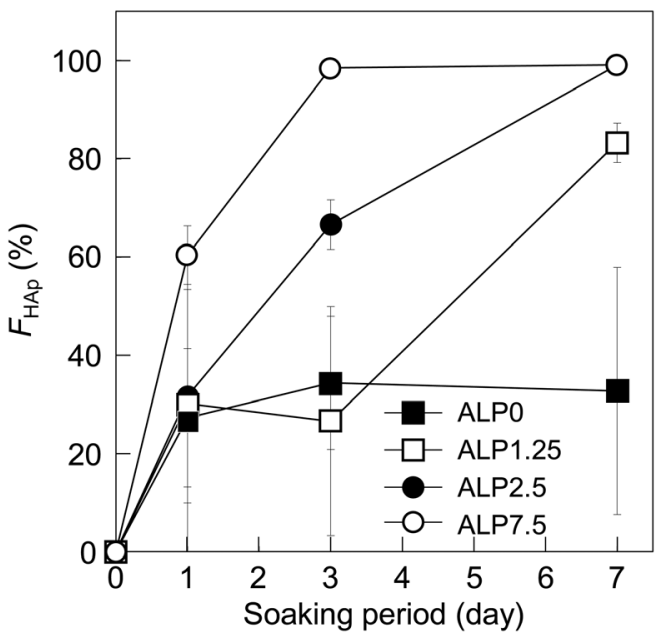

Fig. 7 Time dependent changes of fraction of HAp with respect to CaPP $\left(F_{\mathrm{HAp}}\right)$ in samples.

concentration (Fig. 7). Hence the ALP promoted the transformation from CaPP to HAp in the SBF.

\section{Conclusions}

Inspired by the formation process of HAp in our bones, we believe that SCPEs are good candidates for biomoleculeresponsive ceramic biomaterials in bone repairing applications. The transformation behaviour of CaPP, which was used as a model SCPE, in SBF modified with ALP, was investigated to develop new concept ceramic biomaterials. CaPP crystals had plate-like shape before being dissolved in solution. After soaking in the SBF containing ALP, the morphology of the CaPP crystals had disappeared, and small precipitates were observed under SEM. The transformation from CaPP to HAp in SBF containing ALP was confirmed by XRD and FTIR analyses, while it did not occur without ALP present. The transformation rate increased with higher ALP concentrations. Notably, the ALP-mediated hydrolysis of phenylphosphate to phenol and orthophosphate ions accelerated the transformation of CaPP to HAp, even when the $\mathrm{pH}$ of the SBF was outside the optimum conditions for ALP activity. The findings in this study demonstrate that CaPP responds to ALP and transforms into HAp. Although not verified in this study, this reaction is also believed to occur in vivo based on the use of the ALP present in our bones. Hence, SCPEs can act as bioresponsive ceramic biomaterials. This concept of developing bioresponsive ceramic materials breaks from the traditional framework of bioceramics and will contribute to the development of novel ceramic biomaterials, including but not limited to the growth of artificial bone. In the future, we will evaluate the toxicity of CaPP by performing cell culture tests and clarify its usefulness for artificial bone growth by performing in vivo experiments.

\section{Conflicts of interest}

There are no conflicts to declare.

\section{Acknowledgements}

This work was partially supported by a Grant-in-Aid for Scientific Research (No. 22107007; C. O.) on Innovative Areas of "Fusion Materials" (No. 2206) and JSPS KAKENHI (No. 18K14308; T. Y.) from the Ministry of Education, Culture, Sports, Science, and Technology, Japan.

\section{References}

1 S. M. Best, A. E. Porter, E. S. Thian and J. Huang, J. Eur. Ceram. Soc., 2008, 28, 1319-1327.

2 N. Tamai, A. Myoui, T. Tomita, T. Nakase, J. Tanaka, T. Ochi and H. Yoshikawa, J. Biomed. Mater. Res., 2002, 59, 110-117.

3 T. Okuda, K. Ioku, I. Yonezawa, H. Minagi, Y. Gonda, G. Kawachi, M. Kamitakahara, Y. Shibata, H. Murayama, H. Kurosawa and T. Ikeda, Biomaterials, 2008, 29, 2719-2728.

4 S. Deville, Materials, 2010, 3, 1913-1927.

5 N. Patel, S. M. Best, W. Bonfield, I. R. Gibson, K. A. Hing, E. Damien and P. A. Revell, J. Mater. Sci.: Mater. Med., 2002, 13, 1199-1206.

6 A. E. Porter, N. Patel, J. N. Skepper, S. M. Best and W. Bonfield, Biomaterials, 2003, 24, 4609-4620.

7 A. E. Porter, C. M. Botelho, M. A. Lopes, J. D. Santos, S. M. Best and W. Bonfield, J. Biomed. Mater. Res., Part A, 2004, 69A, 670-679.

8 C. L. Camiré, S. J. Saint-Jean, C. Mochales, P. Nevsten, J. S. Wang, L. Lidgren, I. McCarthy and M. P. Ginebra, J. Biomed. Mater. Res., Part B, 2006, 76B, 424-431.

9 J. W. Reid, L. Tuck, M. Sayer, K. Fargo and J. A. Hendry, Biomaterials, 2006, 27, 2916-2925.

10 H. Orimo, J. Nippon Med. Sch., 2010, 77, 4-12.

11 K. Yamauchi, T. Goda, N. Takeuchi, H. Einaga and T. Tanabe, Biomaterials, 2004, 25, 5481-5489.

12 Y. Takenaka, M. Iijima, S. Kawano, Y. Akita, T. Yoshida, Y. Doi and I. Sekine, J. Ceram. Soc. Jpn., 2008, 116, 92-95.

13 H. Tanaka and D. Ihata, Mater. Res. Bull., 2010, 45, 103-108. 14 M. Xie, M. Ø. Olderøy, Z. Zhang, J. P. Andreassen, B. L. Strand and P. Sikorski, RSC Adv., 2012, 2, 1457-1465.

15 T. E. L. Douglas, A. Piegat, H. A. Declercq, D. Schaubroeck, L. Balcaen, V. Bliznuk, B. D. Meyer, F. Vanhaecke, R. Cornelissen, M. E. Fray and P. Dubruel, Mater. Lett., 2014, 137, 62-67.

16 T. Kokubo, H. Kushitani, S. Sakka, T. Kitsugi and T. Yamamuro, J. Biomed. Mater. Res., 1990, 24, 721-734.

17 U. Sharma, D. Pal and R. Prasad, Indian J. Clin. Biochem., 2014, 29, 269-278.

18 H. Tanaka, K. Ibaraki, M. Uemura, R. Hino, K. Kandori and

T. Ishikawa, Mater. Res. Bull., 2007, 42, 1364-1373.

19 S. Koutsopoulos, J. Biomed. Mater. Res., 2002, 62, 600-612. 